\title{
Tritium Behavior in Eroded Dust and Debris of Plasma-Facing Materials*
}

\author{
RECEATED \\ SEP 211999 \\ A. Hassanein ET O.STI \\ Argonne National Laboratory, Argonne, II 60439, USA
}

B. Wiechers

Forschungszentrum Jülich GmbH, D-52425 Jülich, Germany

\section{Konkashbaev}

Troitsk Institute for Innovation and Fusion Research, Troitsk, Russia

January 1998

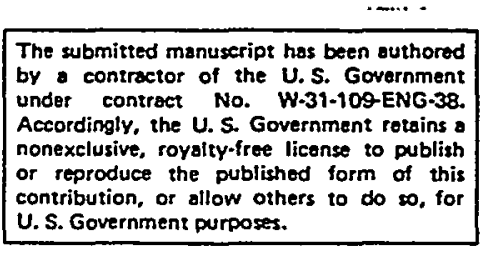

Presented at the Eighth International Conference on Fusion Reactor Materials (ICFRM-8) October 26-31, 1997, Sendai, Japan.

"Work supported by the U.S. Department of Energy, and by the Ministry of Atomic Energy and Industry, Russia. 


\section{DISCLAIMER}

This report was prepared as an account of work sponsored by an agency of the United States Government. Neither the United States Government nor any agency thereof, nor any of their employees, make any warranty, express or implied, or assumes any legal liability or responsibility for the accuracy, completeness, or usefulness of any information, apparatus, product, or process disclosed, or represents that its use would not infringe privately owned rights. Reference herein to any specific commercial product, process, or service by trade name, trademark, manufacturer, or otherwise does not necessarily constitute or imply its endorsement, recommendation, or favoring by the United States Government or any agency thereof. The views and opinions of authors expressed herein do not necessarily state or reflect those of the United States Government or any agency thereof. 


\section{DISCLAIMER}

Portions of this document may be illegible in electronic image products. Images are produced from the best available original document. 


\title{
Tritium Behavior in Eroded Dust and Debris of Plasma-Facing Materials
}

\author{
A. Hassanein' ${ }^{1}$ B. Wiechers ${ }^{2}$, and I. Konkashbaev ${ }^{3}$ \\ 'Argonne National Laboratory, Argonne, Il 60439, USA \\ ${ }^{2}$ Forschungszentrum Jülich GmbH, D-52425 Jülich, Germany \\ ${ }^{3}$ Troitsk Institute for Innovation and Fusion Research, Troitsk, Russia
}

Tritium behavior in plasma-facing components (PFCs) of future tokamak reactors such as ITER is an essential factor in evaluating and choosing the ideal plasma-facing materials (PFMs). One important parameter that influences tritium buildup and release in candidate materials is the effect of material porosity on tritium diffusion and retention. Diffusion in porous materials, for example, consists of three different processes: along grain boundaries, along microcrystallite boundaries, and in pure crystallite structures. Such diffusion processes have strong nonlinear behavior due to temperature, solubility, and existing trap sites. Therefore, a realistic model for tritium diffusion in porous and neutronirradiated materials must account for both nonlinear and multidimensional effects. A tritium transport computer model, TRAPS, has been developed to evaluate and predict the kinetics of tritium transport in porous media. This two-dimensional model incorporates tritium diffusion and trapping processes that also account for hydrogen-isotope solubility limits in PFMs. This model is being coupled with the computer model, TRICS, which has been developed to study the effect of surface erosion on tritium behavior in PFCs. 


\section{Introduction}

Hydrogen isotope trapping and release by plasma-facing materials (PFMs) will control fuel retention and recycling in future fusion reactors. An understanding of particle and energy flow between PFMs and plasma is also necessary for optimizing plasma performance and for ensuring safe and reliable reactor operation. Studies of hydrogen diffusion and retention in fusion reactor materials have been carried out extensively over recent years [1-5]. An important parameter that influences tritium buildup and release in candidate reactor materials is the effect of material porosity on the diffusion and retention of hydrogen isotopes. Figure 1 schematically illustrates the structure of porous materials. Diffusion in porous materials consists of three different and distinct processes: along grain boundaries, along microcrystallite boundaries, and in pure crystallite structures. However, a comprehensive and reliable model of these phenomena does not exist because both the experimental and theoretical results of the retention and transport of hydrogen isotopes in PFM often contradict each other [2]. In particular, this discrepancy occurs because diffusivity in real candidate materials (graphites, Be, W) is much greater (several orders of magnitude) than that of ideal homogeneous crystals; this is very well described by theory [4]. Existing models do not self-consistently account for the strong inhomogeneous structure of real PFMs.

It is well known, for example, that eroded material the divertor PFM can form a redeposited layer. The dust and debris of the eroded PFM may also be redeposited in different chemical forms (for example, $\mathrm{CT}_{4}$ ). Therefore, new materials can be produced during reactor operation. The structure, chemical composition, transport, and retention properties of these new materials are quite different from those of initial PFM. One must also realize that the redeposited or codeposited layers are very porous and inhomogeneous. possibly resulting in more retained tritium than that in the initial PFM. It may seem obvious 
that diffusion and outgassing of the new PFM will occur much faster than in the original PFM. In this report, a new model of diffusion into nonhomogeneous porous materials is developed and implemented in the computer code TRAPS (Tritium Accumulation in Pourous Structure).

\section{Tritium Transport Mechanisms in the 2-D TRAPS Code}

The 2- and 3-dimensional effects of the porous structure of PFMs and the redeposited dust and debris of these materials on tritium diffusion and inventory were previously discussed in detail [5]. Therefore, one very important feature of the developed model is enhanced and presented below, i.e., modeling of diffusion in grain-structured materials. It is known that ITER candidate materials (C, Be, W) will consist of grains with size, $L_{\mathfrak{g}}$, of a few micrometers and separated from each other by an intergranular substrate with width, $d_{b}$, where $d_{g} \approx 0.1 L_{b}$. The grains, in turn, consist of crystallites with size, $L_{c}$, of $\approx 50-100 \AA$ a and that are also separated from each other by intercrystallite substrates with width $d_{c} \approx 0.1 L_{c}$, as schematically illustrated in Fig. 2 .

The diffusion coefficients of both the intergranular substrate, $D_{k}$, and the intercrystallite substrate, $D_{a}$, are much greater than the diffusion coefficient, $D_{c}$, of the crystallites, which is close to that of an ideal crystal. This means that implanted plasma particles (T,D) will diffuse mainly along these substrates. After saturation of the substrates, the implanted gas diffuses into the crystallites. Because of the small size, $\mathrm{L}_{c}$, of the crystallites, the diffusion time into the crystallites, $\tau_{c}$, becomes comparable to or smaller than the diffusion time along the substrates: 


$$
\tau_{c}=\frac{\mathrm{L}_{c}^{2}}{\mathrm{D}_{\mathrm{c}}}=\frac{\mathrm{L}_{\mathrm{g}}^{2}}{\mathrm{D}_{\mathrm{k} / \mathrm{a}}}, \mathrm{L}_{\mathrm{c}} \ll \mathrm{L}_{\mathrm{g}}, \mathrm{D}_{\mathrm{k}}=\mathrm{D}_{\mathrm{a}}
$$

In describing diffusion in such a medium, direct numerical modeling requires extensive computer time. Therefore, we use a set of equations that regard this inhomogeneous discrete system of grains and crystallites as a continuous medium while accounting for the inherent discrete structure, i.e., existence of grains, crystallites, and intergranular/intercrystallite substrates.

The full set of equations describing such a system is very complicated; therefore, for purposes of this work only a simplified set of equations is given, ignoring diffusion in the vertical intercrystallite channels (along the $\mathrm{Y}$ axis) and in the horizontal intergranular channels (along the $\mathrm{X}$ axis). In this model, crystallites are also regarded as spheres with an effective radius close to that of $\mathrm{L}_{c}$. This set of equations is given by

$$
\begin{aligned}
& \frac{\partial \mathrm{n}_{\mathrm{k}}}{\partial \mathrm{t}}=\mathrm{D}_{\mathrm{k}} \cdot \frac{\partial^{2} \mathrm{n}_{\mathrm{k}}}{\partial \mathrm{y}^{2}}-S_{\mathrm{ka}}+S_{\mathrm{ak}}-S_{\mathrm{kt}}^{+}+S_{\mathrm{kt}}^{-} \\
& \frac{\partial \mathrm{n}_{\mathrm{a}}}{\partial \mathrm{t}}=\mathrm{D}_{\mathrm{a}}^{*} \frac{\partial^{2}-\mathrm{n}_{\mathrm{k}}}{\partial \mathrm{x}^{2}}-S_{\mathrm{ac}}+S_{\mathrm{ca}}-S_{\mathrm{at}}^{+}+S_{\mathrm{at}}^{-} \\
& \frac{\partial \mathrm{n}_{\mathrm{c}}}{\partial \mathrm{t}}=\mathrm{D}_{\mathrm{c}}^{*} \frac{1}{\mathrm{r}^{2}} \frac{\partial}{\partial \mathrm{r}} \mathrm{r}^{2} \frac{\partial \mathrm{n}_{\mathrm{c}}}{\partial \mathrm{r}}+S_{\mathrm{ac}}-S_{\mathrm{ct}}^{+}+S_{\mathrm{ct}}^{-}
\end{aligned}
$$

where the subscripts $k, a, c$ are those relating to the intergranular substrate, intercrystallite substrate, and crystallites, respectively. A simple model is used to account for hydrogen isotope solubility in the porous material, in which diffusion is limited in a simple form: 


$$
\begin{aligned}
& D_{k}^{*}=D_{k}\left[1-\frac{n_{k}(y)}{n_{k s}}\right] \\
& D_{u}^{*}=D_{a}\left[1-\frac{n_{u}(y, x)}{n_{a s}}\right] \\
& D_{c}^{*}=D_{c}\left[1-\frac{n_{c}(y, x, r)}{n_{c s}}\right],
\end{aligned}
$$

where $D_{\alpha},(\alpha=k, a, c)$ is the corresponding diffusion coefficient with $I_{\alpha}$ and $\tau_{\alpha}$ are.the corresponding path length and collision time of medium $\alpha$, and $n_{\alpha s}$ is the maximum dissolved density. Fluxes between the different microstructure, taking solubility into account, are given by

$$
\begin{aligned}
& S_{k a}=n_{k}(y)\left[1-\frac{n_{a}(y, 0)}{n_{a s}}\right] \frac{l_{k}}{\tau_{k}} \frac{N_{c} d_{a}}{d_{k}} \\
& S_{a k}=n_{a}(y, 0)\left[1-\frac{n_{k}(y)}{n_{a s}}\right] \frac{l_{a}}{\tau_{a}} \frac{N_{c} d_{a}}{d_{k}} \\
& S_{c a}=n_{c}(y, x, 0)\left[1-\frac{n_{a}(y, x)}{n_{c s}}\right] \frac{1}{\tau_{c}} \\
& S_{a c}=n_{a}(y, x)\left[1-\frac{n_{c}(y, x, 0)}{n_{a s}}\right] \frac{l_{a}}{\tau_{a}}
\end{aligned}
$$

where $N_{c}=\frac{L_{g}}{L_{c}}$ is the linear number of crystallites in one grain. Fluxes into traps, $S_{a l}^{+}$, and out of traps, $S_{\mathrm{at}}^{-}$, are given by the following relations: 


$$
S_{\alpha l}^{+}=\frac{n_{\alpha}}{\tau_{\alpha l}^{+}}, S_{\alpha l}^{-}=\frac{n_{1}}{\tau_{\alpha l}^{-}}, \tau_{\alpha l}^{-}=\tau_{\alpha l_{o}}^{+} e^{-\varphi / T},
$$

where $\tau_{\alpha t}^{+}$and $\tau_{\alpha t}^{-}$are residence times of capture and detrapping of tritium gas, and $\varphi$ is the trap potential. The definite number of traps, $\mathrm{n}_{\text {ats }}$, is taken into account by using the capture time in the following form, which takes into account trap filling:

$$
\tau_{\mathrm{at}}^{+}=\tau_{\mathrm{at0} 0} /\left(1-\frac{\mathrm{n}_{\alpha \mathrm{t}}}{\mathrm{n}_{\alpha \mathrm{st}}}\right)^{2 / 3}
$$

Results of such modeling indicate three distinctive regimes with three effective diffusion coefficients $D_{1}, D_{2}, D_{3}$, corresponding to diffusion along grain boundaries, along crystallites boundaries, and into crystallites, and accounting for the 2-dimensional structure of the channels. The analysis indicates strong dependence on the porous structure parameters and dimensions. Figure 3, for example, shows the gas density distribution along one intergranular channel for the following parameters:

$$
\begin{aligned}
& \mathrm{D}_{\mathrm{k}} / \mathrm{D}_{\mathrm{c}}=\dot{\mathrm{D}}_{\mathrm{a}} / \mathrm{D}_{\mathrm{c}}=100, \mathrm{~L}_{\mathrm{g}}=1 \mu \mathrm{m}, \mathrm{L}_{\mathrm{c}}=100 \AA \\
& \mathrm{n}_{\mathrm{ks}}=\mathrm{n}_{\mathrm{as}}=\mathrm{n}_{\mathrm{cs}}=10^{18} \mathrm{~cm}^{-3} \\
& \mathrm{~s}, \mathrm{n}_{\mathrm{k}: \mathrm{s}}=\mathrm{n}_{\mathrm{ats}}=\mathrm{n}_{\mathrm{cts}}=10^{17} \mathrm{~cm}^{-3} .
\end{aligned}
$$

As follows from the calculations, the diffusion front velocity is determined by filling of media to the maximum density, $\left(n_{a s}+n_{\text {ats }}\right)$. In the limiting case, 


$$
\frac{\mathrm{L}_{c}^{2}}{D_{c}} \ll \frac{L_{g}^{2}}{D_{k, a}}, D_{k}=D_{a}=D_{0} .
$$

The set of equations, therefore, reduces to one nonlinear equation given by

$$
\frac{\partial \mathrm{n}}{\partial \mathrm{t}}=\mathrm{D}^{*}\left(1-\frac{\mathrm{n}}{\mathrm{n}_{\max }}\right) \frac{\partial^{2} \mathrm{n}}{\partial \mathrm{y}^{2}}
$$

where $D^{*}$ is the normalized diffusion coefficient:

$$
D^{*}=D_{o} /\left(1+L_{\varepsilon} / d_{k}\right)
$$

Equation 8 , therefore, has a self-similar solution given by:

$$
\mathrm{n}=\mathrm{n}_{\max } \mathrm{f}(\xi), \xi=y / \sqrt{D_{0} t}
$$

with the diffusion front expanding as:

$$
Y_{f}=\sqrt{2 D_{0} t /\left(1+L_{\varepsilon} / L_{c}\right)}
$$

Therefore, the total amount of diffusing particles increases as $N \approx n_{\max } Y_{\mathfrak{f}}$. The solution given in Eq. 10 can thus be used to estimate the permeation and retention of tritium in the porous PFMs. 
To model the behavior of tritium in the original PFM as a result of erosion, a version of the DIFFUSE code [6] was significantly enhanced and modified in the self-consistent computer model TRICS (Tritium In Compound System) which can be coupled to TRAPS code. First, the code was enhanced to include up to four different traps with different spatial and energy distribution in the coating and in the substrate materials. Figure 4 is a schematic illustration of the implanted, recombination, diffusing, and trapped fluxes. The inhomogeneous trap distribution is necessary to account for traps created by the implanted flux, which is near the surface region, and those traps created by neutron irradiation, which are distributed throughout the coating and substrate structures.

Surface erosion in the TRICS code is implemented in two ways. A constant erosion rate is the result of normal operation, and a pulsed erosion rate is due to abnormal events. The constant erosion rate is due mainly to physical and chemical sputtering of the surface coating material; as a result, the surface location is a moving coordinate with time. The surface temperature will continue to decrease because the thickness of the material is decreasing and the incident heat flux is presumed to be time-independent. Figure 5 shows an illustration of such processes. At each time-step, the coating thickness is decreased by a certain amount depending on erosion rate. The decrease in the surface temperature as a result will have two main effects: a reduction in the recombined flux at the surface and, as a result, an increase in the permeating flux on the coolant side. The reduction in coating thickness will also reduce the tritium inventory and the diffusing flux, as shown in Fig. 6 for typical reactor conditions given in Ref. 6 .

The TRICS code also accounts for (sudden) pulsed erosion due to abnormal events such as plasma disruptions [7]. In this case, a surface layer of a certain thickness is removed with all its tritium inventory and trap concentrations. The code can also model tritium behavior in eroded or splashed material, resulting from plasma instabilities, as well 
as in the redeposition of this material on colder nearby surfaces. Future work will include accurate estimates of tritium diffusion and inventory for conditions similar to those in future tokamak devices.

\section{Conclusions}

The effect of the porosity of redeposited dust and debris materials on tritium diffusion and inventory in such materials can be quite important. The 2-and 3-dimensional effects of the porous structure of the redeposited dust and debris of PFMs (carbon, beryllium,...), as well as that from plasma-spraying techniques or from original asfabricated materials, must be taken into account in the numerical models that describe gas diffusion and release in porous two-level structural materials. Specifically designed laboratory experiments in which the dust and debris of candidate PFMs are produced and studied, are required in order to correctly predict tritium behavior in these materials. Preliminary models were developed for diffusion in porous materials, including diffusion along grain boundaries, intercrystallite substrates, and into crystallites. It follows from the TRAPS code calculations that filling of porous materials with certain solubilities and numbers of traps has the form of a "filling-wave" that becomes 1-dimensional for longer times exceeding the characteristic times of diffusion into separate grains and crystallites. The TRAPS code also accounts for other important processes such as recombination rates of atoms in materials and pore surfaces, and existence of open or closed pores. Future analysis will evaluate and then assess the total tritium inventory in porous materials as a function of reactor operating conditions. The TRICS code will be used to study the effect of surface erosion on tritium behavior in the original plasma-facing materials and the initial tritium concentration in the eroded redeposited materials. Erosion generally will increase tritium activities in the coolant and in the eroded and splashed debris due to permeation and trapping. 


\section{Acknowledgments}

The authors thank Dr. L. Nikandrov for helpful discussions. Work supported by the U.S. Department of Energy and by the Ministry of Atomic Energy and Industry, Russia. 


\section{References}

[1] P.L. Andrew, M.A. Pick. J. Nucl. Mater. 212-215, (1994) 111.

[2] G. Federici, R. Causey, P.L. Andrew, C.H. Wu., Fusion Eng. and Design 28, (1995) 136.

[3] M.I. Baskes, J. Nucl. Mater. 85 \& 86, (1980) 318.

[4] I.L. Tazhibaeva et al., "Hydrogen release from neutron irradiated graphite and beryllium," presented at 18th Symp. on Fusion Technology (SOFT), Aug. 22-26, 1996, Karlsruhe, Germany.

[5] I.K. Konkashbaev, A. Hassanein, and Yu.B. Grebenshchikov, "Tritium containment in the dust of plasma-facing material produced during operation," presented at 19th Symp. on Fusion Technology (SOFT), Sept. 16-20, 1996, Lisbon, Portugal.

[6] M.I. Baskes, DIFFUSE 83, Sandia National Laboratories Report, SAND83-8231.

[7] A. Hassanein and I. Konkashbaev, J. Nucl. Mater., 233-237 (1996) 713. 


\section{Figure Captions}

Figure 1 Schematic view of porous structure.

Figure 2 Numerical model of two-level structure.

Figure 3 Tritium density along interrgranular substrate, $t / \tau_{\rho}=10000, \tau_{o}=L_{c}{ }^{2} / D_{0}$.

Figure 4 Schematic illustration of implantation, recombination, diffusing, and trapped fluxes.

Figure 5 Illustration of erosion process and moving surface coordinate.

Figure 6 Tritium inventory as a function of time during constant surface erosion rate. 


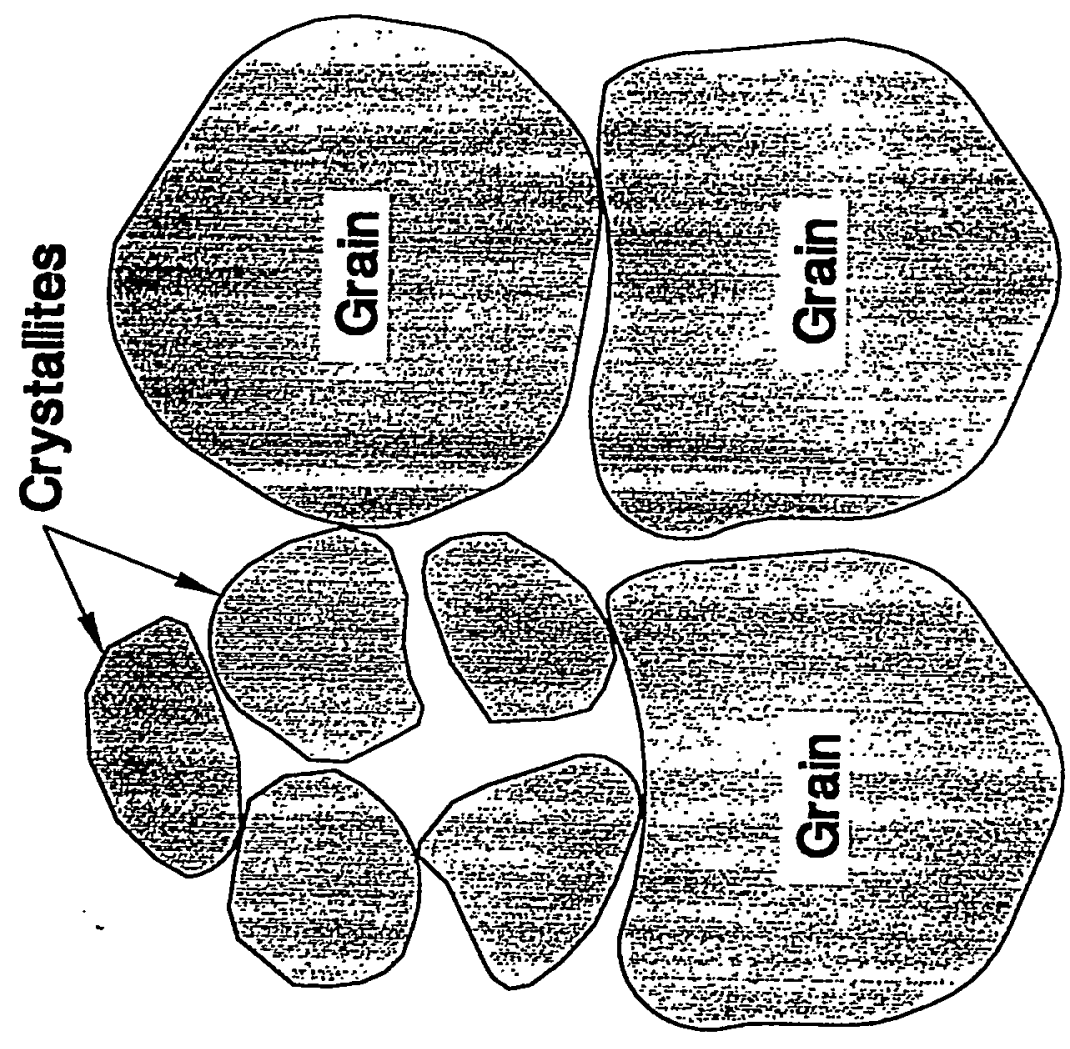

$\theta$ 4

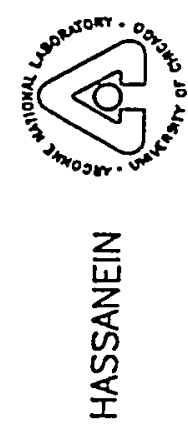




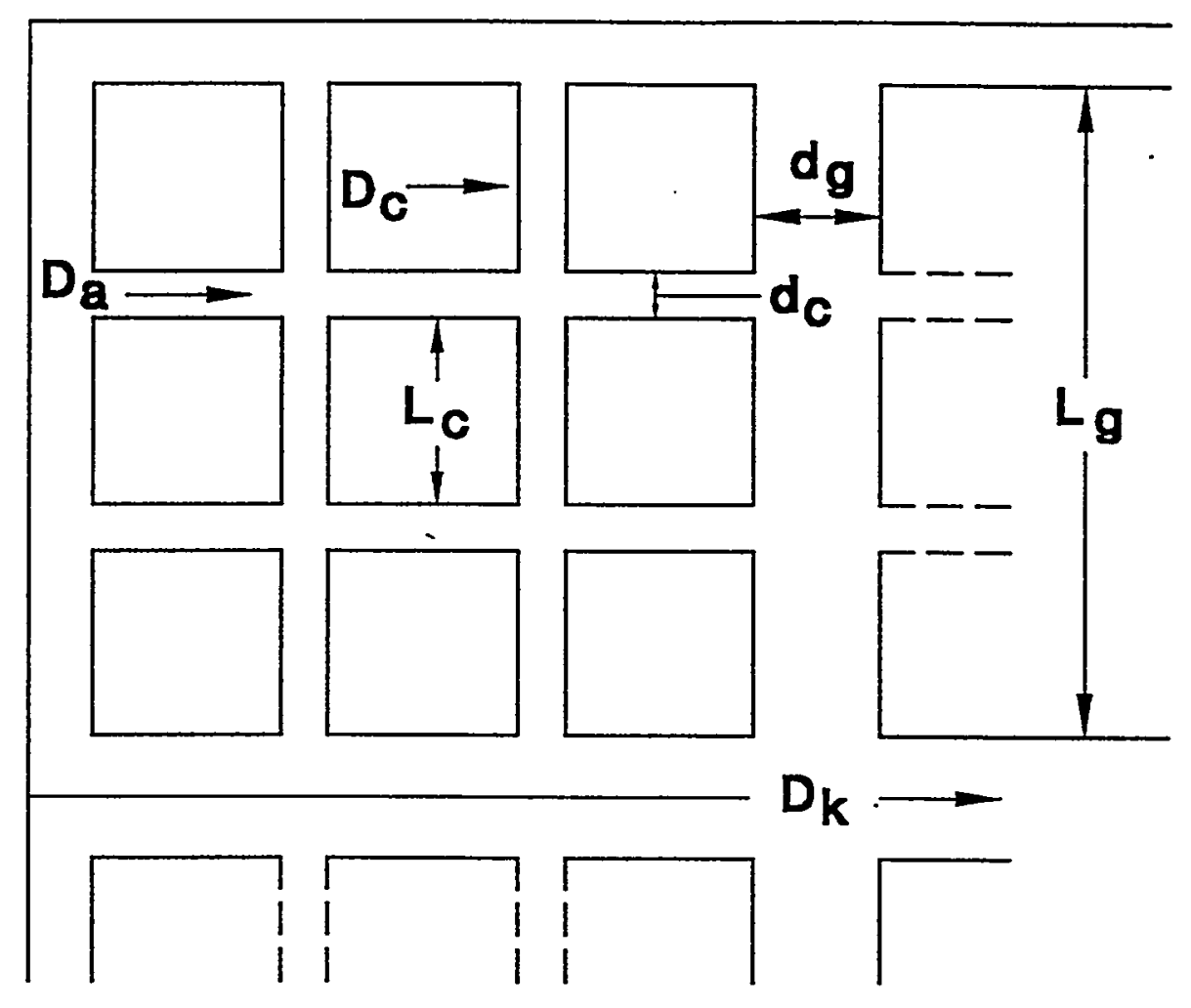

HASSANEIN

Fig (2) 


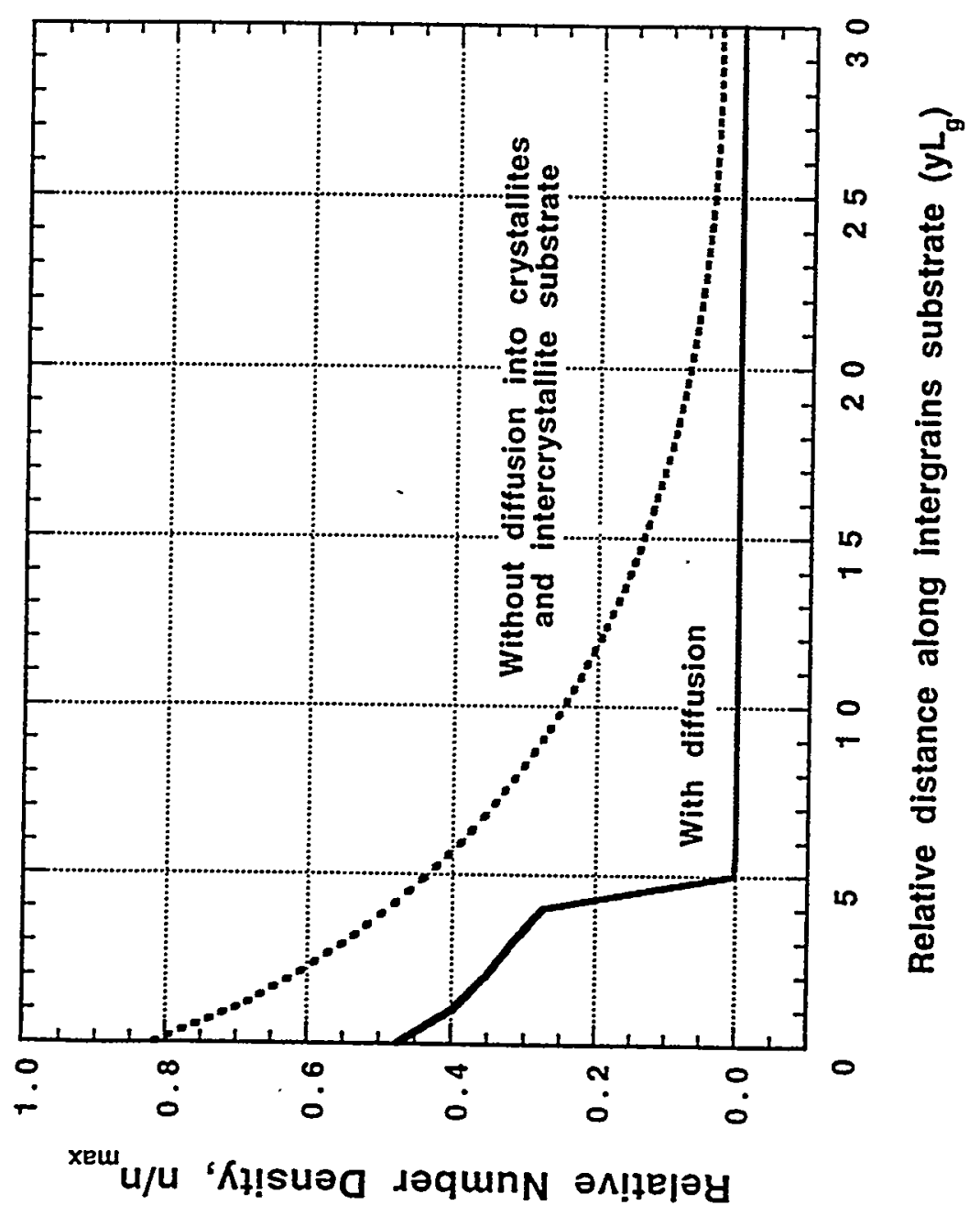

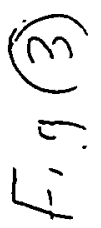




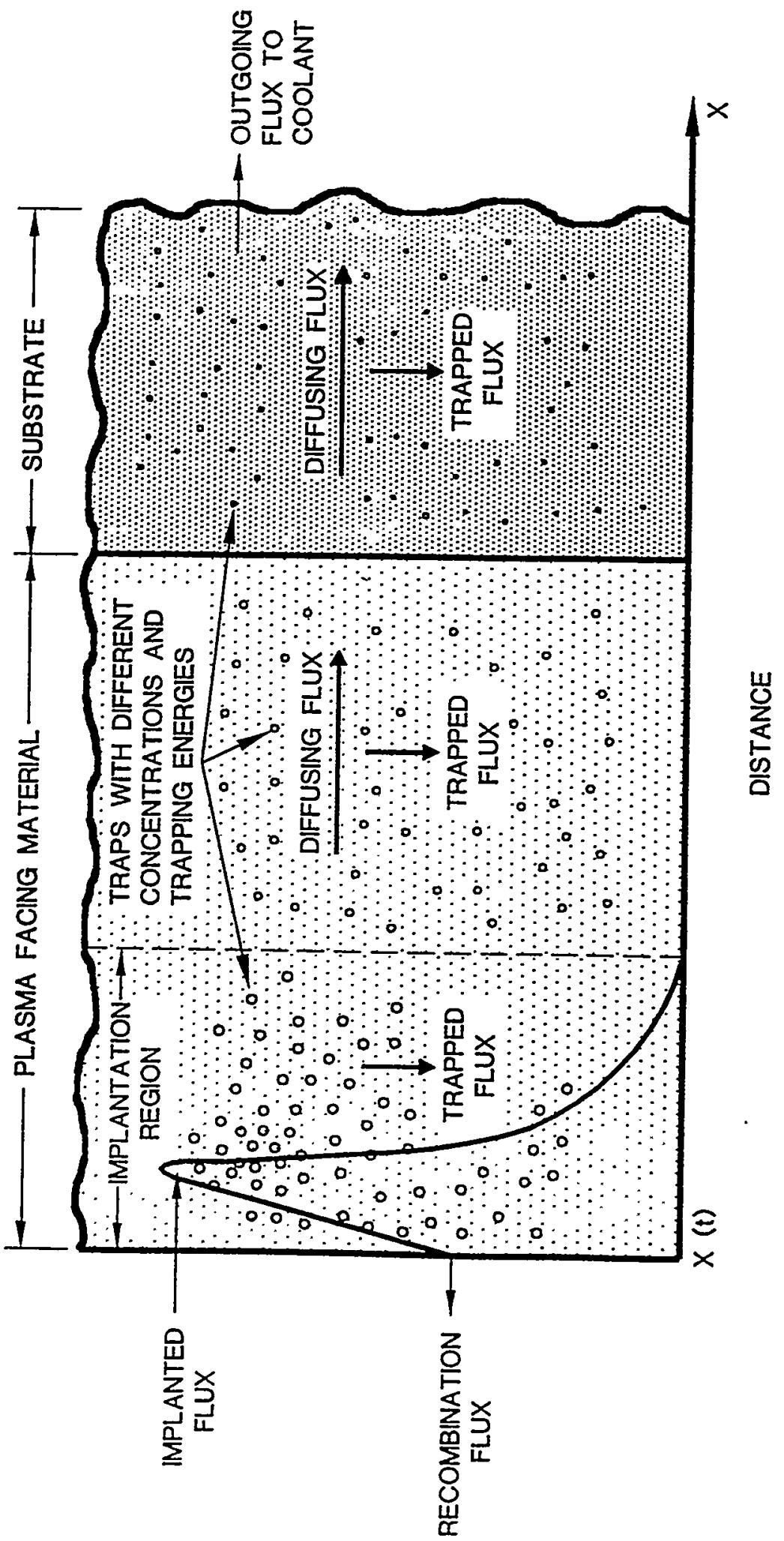

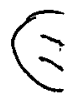




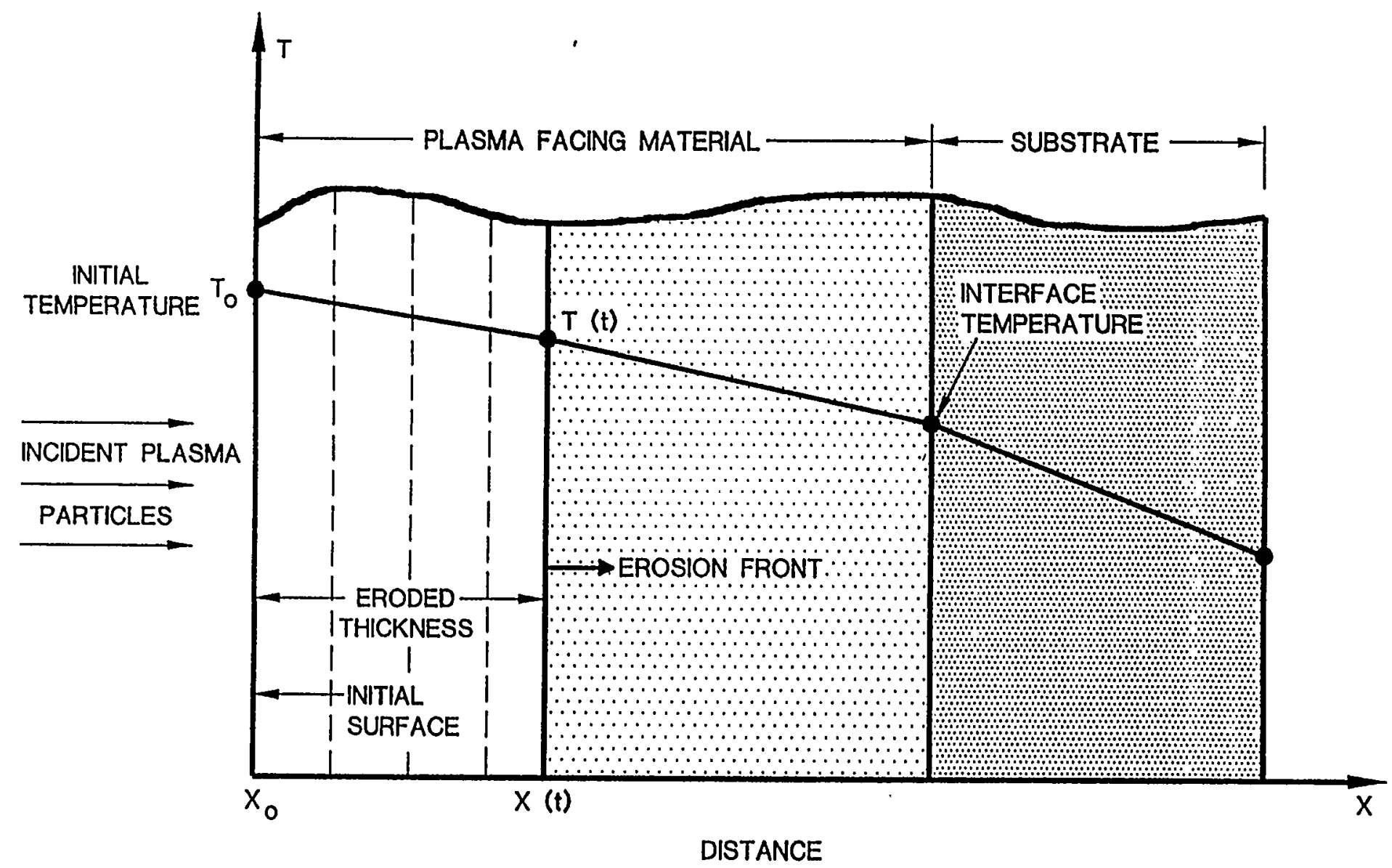

HASSANEIN

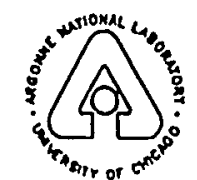




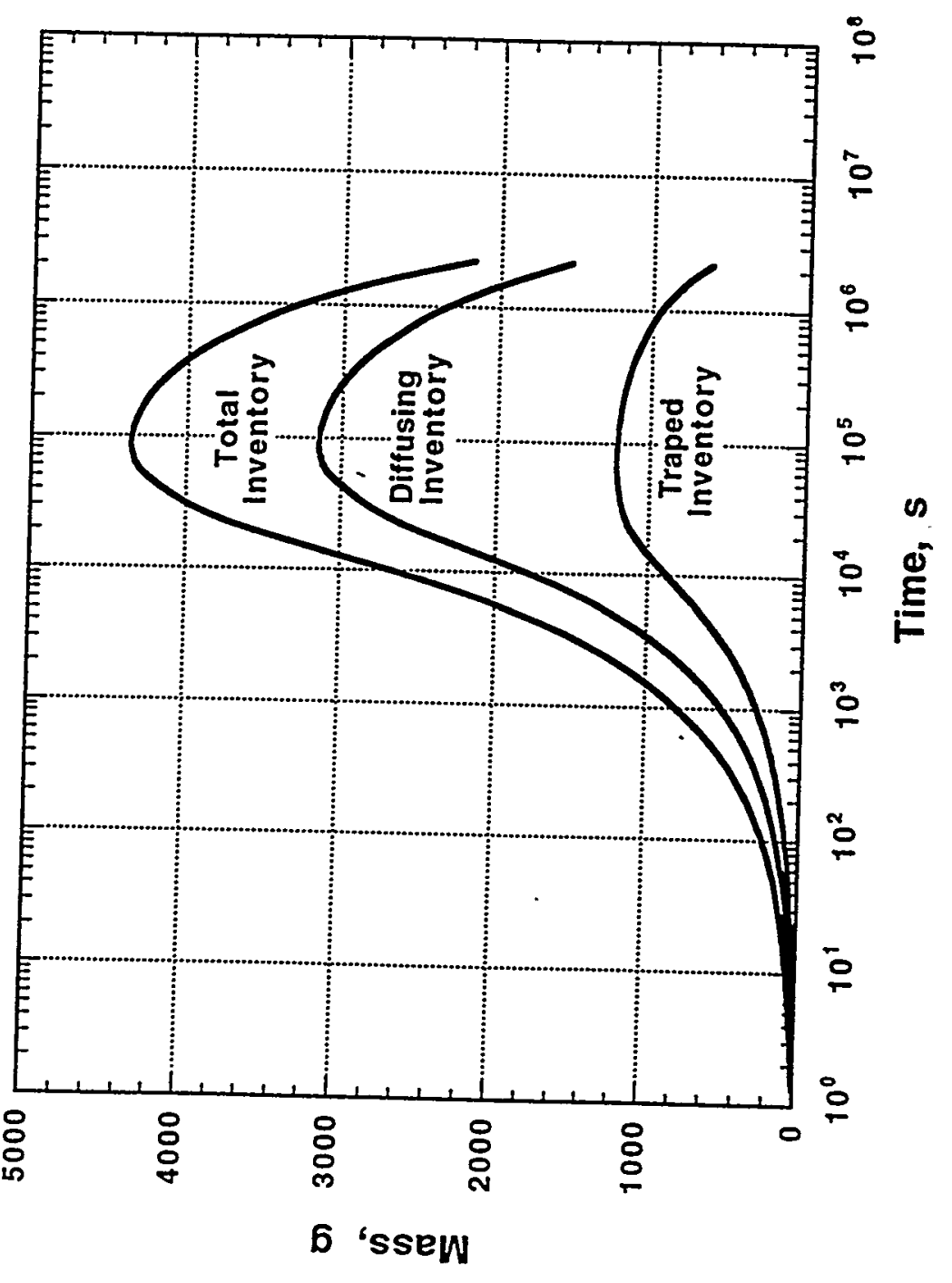

6

5 